\title{
Mechanical impedance control in the human arm while manually transporting an open-top fluid filled dish
}

\author{
Navit Roth ${ }^{\mathrm{a}}$, Rami Seliktar ${ }^{\mathrm{b}}$ and Joseph Mizrahi ${ }^{\mathrm{a}, *}$ \\ ${ }^{a}$ Department of Biomedical Engineering, Technion, Israel Institute of Technology, Haifa, Israel \\ ${ }^{\mathrm{b}}$ School of Biomedical Engineering, Science and Health Systems, Drexel University, Philadelphia, PA, USA
}

\begin{abstract}
The present study deals with stabilizing aspects of a hand-held dish filled with liquid while walking steadily. This is an attempt to decipher the neuro-muscular strategies employed and the mechanical responses of the arm during certain tasks of manual materials handling. The experimental configuration included a cup and the test-subject's hand as an 'end-effector' of a serial three-link system representing the upper limb. These links are connected together by the wrist, elbow and shoulder joints. The tested subjects walked at constant speed on a treadmill while aiming to minimize liquid spillage from the cup. The motion of the limb and shoulder girdle served as inputs to a model to reveal the impedance adjustments during the simultaneous control of grasping and walking under ordinary conditions, and when one of the joints is affected. A regressive function used to express stiffness, included first-order dependence on angle and on angular velocity. The function used for damping included first-order dependence on angular velocity. Redundancies in the numerical solution were eliminated using multicollinearity diagnostic algorithms. The results revealed that the wrist joint was found to have constant stiffness and damping and no regulation of these coefficients was necessary during gait. Both in the elbow and shoulder joints stiffness included a constant coefficient as well as an angular velocity-dependent coefficient. Although all tested subjects demonstrated ability to prevent spillage of liquid, there was a considerable variability among the results obtained, indicating that the compensatory mechanisms employed by each subject to regulate the mechanical impedance were subjective. These results can help in the optimization of manual materials handeling tasks in industrial settings as well as future design of prosthetic arms, robotic appliances and man machine interfacing devices.
\end{abstract}

Keywords: Motor control, joint constraints, haptics, mechanical impedance, prehensile system, balanced walking, manual materials handling

\section{Introduction}

Transporting a cup filled with liquid (e.g. tea, water) is a common daily activity. Clearly, the aim in this task is to navigate the moving hand in such a manner, that chances of spillage of liquid are minimized. While the moving "base" (the torso-shoulder complex) is for the most part translating in a consistent pattern, unintended motion perturbations of the holding hand may occur and their effect on the stability of

${ }^{*}$ Corresponding author: Prof. Joseph Mizrahi, Department of Biomedical Engineering, Technion, Israel Institute of Technology, Haifa 32000, Israel. E-mail: jm@ bm.technion.ac.il. transportation is addressed by corresponding motion control of the joints of the upper limb. The research question of this work is hence, how does the body control these joint movements in order to perform the transportation task?

Mechanically, the upper limb can be represented by three major segments; the hand, the forearm and the upper arm, linked by the shoulder, elbow and wrist joints. The segments are assumed to be rigid bodies, with known dimensions, masses, centers of mass and moments of inertia. Lumped parameters models can be used to describe the dynamic behavior of the joints. In these models, elements of the human body, namely: the muscles, tendons and ligaments, are lumped together 
so that the overall musculoskeletal structure is represented as a damped elastic mechanism. The benefit of lumping is apparent due to the fact that some 32 muscles are involved in positioning of the human hand [23], (Popovic et al., [36]).

The semi repetitive oscillations of the shoulder girdle produced by the ambulatory mechanism in concert with the motion of the joints of the upper limb, navigate the hand holding the cup of liquid in an oscillatory-like motion.

The complex relationship between torque, angular position and angular velocity, termed mechanical impedance, defines the stiffness and damping characteristics of the joint. Controlling the mechanical impedance of the upper limb joints is an important feature of the neuromuscular system which facilitates stabilization of hand-held objects in space, or attenuation of the effect of externally applied forces [26]. It should also be noted that the joint impedance is controlled by the central nervous system through the activation level of the antagonist muscles of the joint. Thus, for a required net torque corresponding to a given joint activity, different levels of contraction of the antagonists will result not only in different impedance levels but also in different energy costs of that activity [8].

Early modeling of the hand-arm system made use of a two degree of freedom representation of that joint with linear impedance to reveal the dynamic response to vibration input to the hand [24]. By also using linear impedance representation, Suggs [27] studied the response of the hand-arm system to vibration and concluded that the hand-arm system may be viewed as a low-pass mechanical filter which attenuates high frequencies. Despite these early results, it has been shown that linear models failed to predict the true dynamic response of joints and that joint impedance is a non-linear phenomenon [7, 12, 21]. Impedance values were also reported to depend on task constraints $[5,6,14,31]$, on the patterns of perturbation and on the actual joint movement configuration [17-19, 29]. Thus, in defining impedance characteristics, stiffness and damping coefficients have been mostly assumed to be variable $[13,33]$.

Past studies related to the combined control of the locomotor and manual handling systems have suggested that locomotion and reaching are closely connected motor activities (Bertram and Marteniuk [1]; van der Wel and Rosenbaum [30]). In a study where the tested subjects were required to pick up an object while walking, Carnahan et al., [2] con- cluded that the motor control system may be organized such that the stability of gait is maintained over variations in the control of grasping. More recently, van der Wel and Rosenbaum [30] studied the coordination of locomotion and prehension while walking by asking subjects to move an object on a tabletop from one position to another. Their findings indicated that the support-leg preferences at grasp time were anticipated by the participants as they walked up to the table. Past studies have also suggested interrelationships between gait and cognitive function and possible existence of a common control mechanism of dynamical processes (Ivanov et al., [9]). This indicated considerable longrange planning of entire body positions associated with forthcoming object transfers. No studies were found dealing with the mechanical aspects of the interaction between grasping and walking level, on the mechanisms of stabilizing the grasped object during walking and on the possible effects due to impairment of one of the joints of the upper limb.

The present study deals with the analysis of motion of the hand grasping a cup filled with liquid while walking. We focused our interest on the non-linear mechanical impedances of the shoulder, elbow and wrist joints while navigating the hand in space so as to avoid or minimize liquid spillage or dripping from the cup. In order to explore the relative role of each of the joint mechanical impedances, different joint disabilities were simulated by the successive immobilization of the shoulder, elbow and wrist joints.

The present study is thus intended to provide an insight into the mechanisms, by which the stiffness and damping are adjusted to accommodate changes taking place during manual transport of objects while walking, so as to ensure stability of the held object. Since impedance-based control strategies require information on the continuous nonlinear behavior of the joints, the results of the present research should pertain among other applications, to the design of spring based artificial and robotic arms.

\section{Methods}

\subsection{Experimental procedure}

\subsubsection{Walking trials while holding 'cup of tea'}

Four female subjects, aged 28-57 (average 35.5, SD 14.3) walked on a treadmill (Woodway PPS55-Med) at a constant speed of $1.25 \mathrm{~m} / \mathrm{s}$ while holding in their 
right hand a cup filled with liquid and fixing their gaze on a distant mark in front of them at eye level. All the subjects were in an excellent state of health, with no previous histories of muscle weakness, neurological diseases or drug therapy. Each subject provided informed consent to participate in the study according to the University's Institutional Review Board's guidelines. The subjects were instructed to keep their right fore-arm and hand moving in the sagittal plane, as stable as possible, so as to maintain the liquid surface level, and to refrain from "liquid spillage". "Liquid spillage" was quantified by using a specially constructed apparatus, as described at a later Section.

The walking tests, each of duration of 30 seconds, were performed under the following conditions: unrestrained joints of the upper limbs, followed by the successively restraining the right wrist, elbow and shoulder joints. Joint restraints were administered by immobilizing each joint around a given flexion angle (wrist $180^{\circ}$, elbow $90^{\circ}$ and shoulder $0^{\circ}$ ). Nearcomplete imobilizations were achieved by means of specially constructed orthotic devices. Tests were repeated five times at each setting and a resting period of 2 min was allowed between the tests.

The following independent variables were recorded for each test:

Kinematics and the joint angles of the right upperlimb in the sagittal plane. Goniometers were used to record the angles of the elbow and wrist joints. A video camera monitored the sagittal motion of two markers attached to the right arm for determination of the angle of the arm relative to the vertical.

Walking cycle; An accelerometer firmly fastened to the right shank near the tibial tuberosity so as to monitor the longitudinal shank acceleration. This acceleration signal was used as event marker to identify heel-strike and synchronize the walking cycle and the measured variables in the upper limb.

Voltage signals indicating the level of liquid tilt within the cup served for monitoring the stability of the liquid level while walking and as a simulated "liquid spillage".

\subsection{Instrumentation}

\subsubsection{Kinematics}

Joint angles served as inputs for the model. The variabily of the joint angle patterns vs. joint restrictions between the subjects were also studied. Goniometers (Biometrics Ltd, Gwent, UK) were used for measure- ments of elbow and wrist angles at the sampling rate of $100 \mathrm{~Hz}$. For kinematic measurements in the sagittal plane two-retroflective hemispherical markers of $2 \mathrm{~cm}$ diameter were used. The markers were located in the following sites: The upper marker was attached to the shoulder joint and the lower one $3 \mathrm{~cm}$ above the elbow joint. Video data were collected using a GRDVL JVC camera at 60 frames/s. The front plane of a cubic calibration cage, each side measuring $1.0 \mathrm{~m}$, was positioned on the treadmill in the plane of motion (sagittal plane) and calibration was made using six control points by means of Direct Linear Transformation (DLT) software. The optical axis of the camera was set centrally and perpendicular to the plane of motion.

The cut off frequency for the kinematic measurements was $3 \mathrm{~Hz}$ for the elbow and wrist joints and $5 \mathrm{~Hz}$ for the shoulder joint. The shoulder angle was calculated from the camera data.

\subsubsection{Accelerometer}

A light-weight (4.2 grams) accelerometer (Kistler PiezoBeam, type 8634B50), connected to a coupler (Kistler Piezotron, type 5122) was used. The accelerometer was pressed onto the skin by a metal holder weighing 1.4 grams in a closest proximity to the bony prominence of the tibial tuberosity of the right leg by means of an elastic band around the shank. It was aligned with the sensitive axis in the direction of the longitudinal axis of the tibia so as to provide the axial component of the acceleration of the shank.

The signals from the accelerometer were fed to the PC-based data acquisition system at a sampling rate of $1000 \mathrm{~Hz}$. A high sampling rate was required to identify the timings of the peak acceleration associated with the foot strike event.

\subsubsection{Quantification of the "liquid spillage"}

An instrumented cup was designed to monitor liquid level (tilt) within the cup as follows. The plastic cup was wired at its inner surface by gluing three paralel transverse circular conductive bands, to mark three different levels of the liquid. The levels corresponded to 70,80 and $90 \%$ of the cup height for the lowest, medium and highest stripe, respectively. Each of these stripes was connected to an electric circuit which was shorted whenever the electrolitic fluid (salted water) was tilted and made contact as a result of ulstable motion event. The initial liquid level within the cup at the rest position was at $60 \%$ of the cup's height. The 
electric signal amplitude was modulated to correspond to stripe height, and served as tilt (or spillage) indicator. These electric signals were sampled at $1000 \mathrm{~Hz}$. Prior to each testing sequence, the electric contacts were examined and the cup fluid level measuring system was calibrated. The fluid tilt data was then filtered at $10 \mathrm{~Hz}$. For further processing. The data obtained from each experiment included the number of times the liquid level exceeded each of the stripes, as well as the liquid spillage volume obtained by integration of the tilt data vs. time.

\subsection{Model}

\subsubsection{Biomechanical model}

A two-dimensional sagittal model consisting of three segments: upper-arm, forearm and hand, describes our system (Fig. 1). The input to the system is the body movement due to walking, as transmitted to the upper limb through the gleno-humeral joint of the shoulder, and the output of the system is the kinematics of the hand holding the fluid filled cup. The arm model segments are assumed to be rigid bodies, with known masses, dimensions, centers of mass and moments of inertia. The joint angles are depicted in Fig. 1. The shoulder angle $\emptyset_{\mathrm{s}}$ is the angle between the upper arm and the vertical. The model segments are connected together by the joints via lumped parameter impedances representing damped springs.

The spring coefficients are expressible in terms of joint angles and angular velocities. Thus,

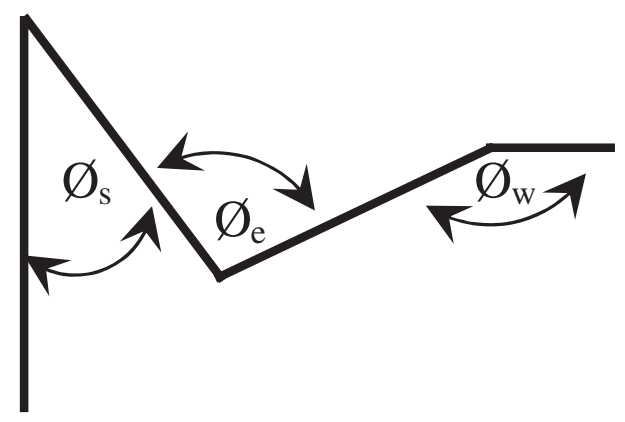

Fig. 1. Sagittal view of segments and joints of the upper limb: $\mathrm{A}=$ upper arm, in relation of vertical axis of the body representing the walking body; $\mathrm{B}=$ forearm; $\mathrm{C}=$ hand. The input to the system is the movement of the walking body, as transmitted to the upper limb through the shoulder girdle and the output of the system is the navigated free hand holding the cup-of-liquid.

$$
k_{j}(\phi)=k_{0 j}+k_{1 j}\left(\phi_{j}-\phi_{0 j}\right)+k_{2 j}\left(\dot{\phi}_{j}-\dot{\phi}_{0 j}\right)
$$

where $k_{j}(\phi)-$ is the stiffness of joint $j$

$$
b_{j}(\dot{\phi})=b_{0 j}+b_{1 j}\left(\dot{\phi}_{j}-\dot{\phi}_{0 j}\right)
$$

where $b_{j}(\dot{\phi})$ - damping of joint $j$

The reference angle $\phi_{0 j}$ was taken in the neutral position of each joint. These coefficients are related to joint torques as follows:

$$
\begin{aligned}
& k_{j}=\frac{\partial M_{j}}{\partial \phi_{j}} \\
& b_{j}=\frac{\partial M_{j}}{\partial \dot{\phi}_{j}}
\end{aligned}
$$

The joint torque is obtainable by integration and by summing up the elastic and damping torques.

$$
\begin{aligned}
M_{t j}= & M_{s t j}+M_{b t j} \\
= & k_{0 j}\left(\phi_{t j}-\phi_{0 j}\right)+\frac{k_{1 j}}{2}\left(\phi_{t j}-\phi_{0 j}\right)^{2} \\
& +k_{2 j}\left[\left(\dot{\phi}_{t j}-\dot{\phi}_{0 j}\right)\left(\phi_{t j}-\phi_{0 j}\right)\right] \\
& +b_{0 j}\left(\dot{\phi}_{t j}-\dot{\phi}_{0 j}\right)+\frac{b_{1 j}}{2}\left(\dot{\phi}_{t j}-\dot{\phi}_{0 j}\right)^{2}
\end{aligned}
$$

\subsubsection{Dynamic model}

The torques $\tau_{j i}$ of the wrist, elbow and shoulder joints were obtained by solving the inverse dynamics for the upper limb using Kane's method [10]. These torques were subsequently used for the calculation of the stiffness and damping coefficients at each joint.

\subsubsection{Parameter estimation}

The stiffness and damping coefficients in Eqs. (5) can be resolved from the calculated torques $\left(\tau_{j} i\right)$ in the dynamic model, by parameter estimation using optimization procedures. Minimization of the following objective function $J_{j}$ was thus performed for each of the joints (wrist, elbow and shoulder).

$$
J_{j}=\sum_{i=1}^{n}\left(\tau_{j i}-M_{j i}\right)^{2}
$$

Here $j$ designates the joint and $n$ is the number of samples of the torque vector. 


\subsubsection{Model constraints}

The following constraints were applied in the estimation process of the model:

(a) Positive values of the overall stiffness (Equation 1), i.e. opposite directions of force and deformation; (b) Positive values of the overall damping (Eq. 2); (c) Positive energy in the spring element; (d) Negative energy in the damping element

We compared the results with and without constraint (a) and found no significant difference.

\subsubsection{Parameter estimation and statistical analysis}

Parameter estimation was performed by using linear least square procedure. Comparison between the various testing conditions was carried out by using $\mathrm{T}$ test for repeated measures and statistical significance was established at $p<0.05$. The results are presented by their means and SD's.

\subsubsection{Multicollinearity diagnostic criteria and reduction of the model}

Parameter identification was made to reveal the joint impedance model which best fits all the tests made with and without joint restrictions, namely whether the general impedance expressions could be reduced to a simpler form. To ensure correct parameter estimation, all predictor variables in the multiple linear regression analysis must be uncorrelated and the model parameters should be independent of each other. Multicollinearity diagnostic criteria combined with $F$-test were used to reveal dependencies and eliminate redundancies in the numerical solution of the stiffness and damping coefficients and reduce the variables in the stiffness and damping functions. A high ratio between the largest eigenvalue to the smallest one was used as a diagnostic criterion to indicate multiple collinearity. Elimination of multicollinearity was achieved by deletion of the offending predictor variable from the regression model, without impairing the ability to predict the system's response. This was administrated by using an iterative process. The $F$-test served to examine the significance of improvement of the sum of the squares of the errors (SSE), used here as a target function. The reduction procedure of the basic model was made separately for each joint.

Sum of the squares of the errors (SSE) was also used for verification of the accuracy of the obtained impedance model in predicting the limb motion behavior under the actual testing conditions.

\subsubsection{Sensitivity analysis}

Sensitivity of the model was tested by evaluating the effect of variation by $5,10,15$ and $20 \%$ on the estimated results and SSE values of each of the following parameters: measured angles, mass and moment of inertia of each segment and the $x$ and $y$ position of the shoulder joint.

\section{Results}

\subsection{Cup level}

In $96 \%$ of the walking experiments the liquid did not reach the second level. In none of the walking experiments did the liquid reach the third, highest, level. This indicates that the task of minimal spillage of the liquid was maintained in the vast majority of the tests.

\subsection{Joints angles}

\subsubsection{Postural angle averages}

Figure 2 shows typical traces of the upper limb joint angles while walking at the speed of $1.25 \mathrm{~m} / \mathrm{s}$ and holding the cup. The traces shown are for the shoulder, elbow and wrist angles (as defined in Fig. 1). Also shown are traces of the horizontal $(x)$ and vertical $(y)$ positions of the shoulder girdle. The dark dots designate heel-strike events of the right foot. Each cycle includes two heel strikes, one for each foot, with about $1 \mathrm{~s}$ interval between them. The $y$ position trace of the shoulder demonstrates the double peak cycles.

Table 1 summarizes the average postural angles for each of the joints, without and with joint restrictions. The values presented are averages of 5 tests, each over the period of $30 \mathrm{~s}$. In the unrestricted case it can be noticed that in the wrist and elbow joints the selected posture was nearly similar in the 4 subjects. In the shoulder angle, however, in three subjects the average arm angle was ahead of the body vertical line (Fig. 1), while in one subject (Number 2) the average postural angle was behind the body vertical line, with the noticeable angle of $\sim 21.5$ degs.

Wrist constraint was of around $180 \mathrm{deg}$ in 3 out of the 4 subjects. In all the 4 subjects the STD of the wrist angle during movement was smaller compared to no constraint. Noteworthy is the fact that in subject num- 

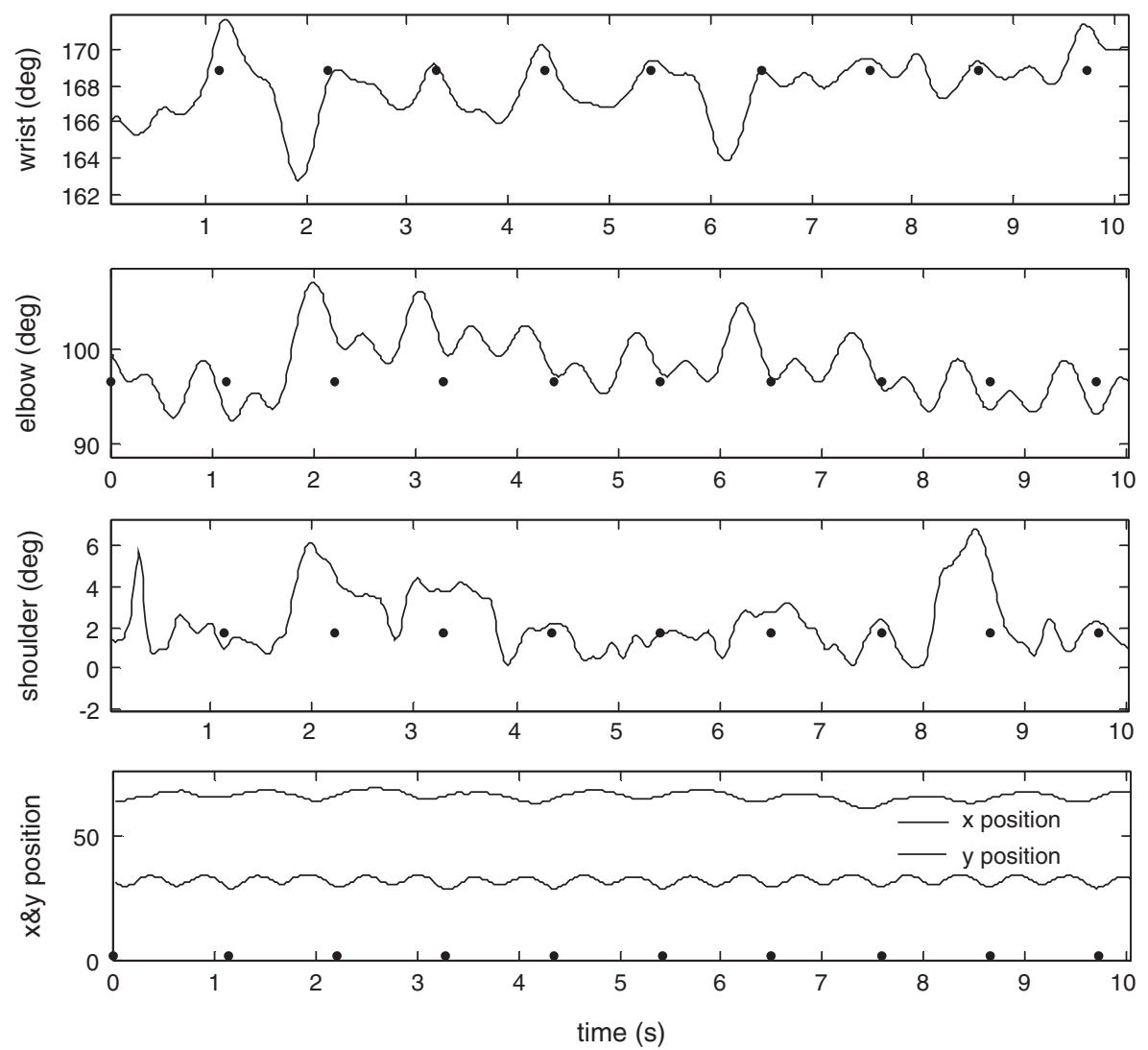

Fig. 2. Typical traces of the joint angles of the upper limb and of the anteroposterior $(x)$ and vertical $(y)$ positions of the shoulder while walking at the speed of $1.25 \mathrm{~m} / \mathrm{s}$ and holding the cup of liquid.

Table 1

Summary of average postural angles for each of the joints, without and with joint restrictions. The values presented are averages of 5 tests, each over the period of $30 \mathrm{~s}$

\begin{tabular}{lcccr}
\hline Restriction & Subject no. & Wrist & Elbow & Shoulder \\
\hline no & 1 & $167.84(1.06)$ & $96.37(1.05)$ & $4.79(2.02)$ \\
& 2 & $164.25(0.84)$ & $67.61(1.50)$ & $-21.51(1.40)$ \\
\multirow{4}{*}{ wrist } & 3 & $164.52(2.84)$ & $80.84(2.65)$ & $6.69(3.50)$ \\
& 4 & $176.20(0.15)$ & $85.67(1.59)$ & $14.98(0.98)$ \\
& 1 & $178.86(0.48)^{*}$ & $93.94(1.84)$ & $4.50(0.84)$ \\
elbow & 2 & $157.57(0.54)^{*}$ & $58.00(1.61)^{*}$ & $-8.20(1.19)^{*}$ \\
& 3 & $177.73(0.37)^{*}$ & $74.77(2.28)^{*}$ & $3.54(0.91)$ \\
& 4 & $179.78(0.11)^{*}$ & $81.78(1.79)^{*}$ & $16.75(0.65)^{*}$ \\
shoulder & 1 & $164.33(1.05)^{*}$ & $126.99(2.03)^{*}$ & $6.58(2.04)$ \\
& 2 & $161.25(1.44)^{*}$ & $101.92(1.30)^{*}$ & $-6.38(2.12)^{*}$ \\
& 3 & $157.92(1.58)^{*}$ & $94.50(0.97)^{*}$ & $3.41(0.88)$ \\
& 4 & $170.75(3.612)^{*}$ & $107.99(1.10)^{*}$ & $17.95(0.80)^{*}$ \\
& 1 & $165.70(2.18)$ & $112.09(1.99)^{*}$ & $3.59(2.42)$ \\
& 2 & $176.47(1.53)^{*}$ & $69.11(2.77)$ & $-10.02(4.25)^{*}$ \\
& 3 & $163.09(0.62)$ & $65.20(1.65)^{*}$ & $2.53(0.80)^{*}$ \\
\hline
\end{tabular}

$* p<0.05$. 
ber 2 the opposite posturing of the forearm necessitated a wrist angle smaller than $180^{\circ}$. Compared to unconstraint, stabilization of the hand under wrist constraint necessitated a decrease in the elbow angle $(p<0.05)$ for all subjects and in the shoulder $(p<0.08)$ for 3 out of the 4 subjects.

Elbow constraint ranged from 94 to $127^{\circ}$. Compared to no constraint, the elbow joint constraint caused a decrease in wrist angle in all subjects $(p<0.01)$. The effect on shoulder joint of elbow constraint was not consistent. Shoulder constraint was aimed at posturing the shoulder joint close at the zero degree angle. The effect of this joint restriction on the postural angles of the other joints was not uniform, indicating that each subject adapted in her individual way to the shoulder restriction.

\subsubsection{Angle amplitudes}

Summary of the average angle amplitudes (deg) for each of the joints, without and with joint restrictions

Table 2

Summary of average amplitude angles (deg) for each of the joints, without and with joint restrictions. The values presented are averages of 5 tests, each over the period of $30 \mathrm{~s}$. The 'no restriction' case served as a reference

\begin{tabular}{lcccc}
\hline Restriction $\backslash$ joint & Subject no & Wrist & Elbow & Shoulder \\
\hline no & 1 & $3.04(0.57)$ & $5.45(0.42)$ & $3.71(0.61)$ \\
& 2 & $1.29(0.31)$ & $2.48(0.40)$ & $3.75(0.29)$ \\
& 3 & $1.26(0.13)$ & $1.70(0.32)$ & $3.92(0.35)$ \\
wrist & 4 & $1.45(0.25)$ & $3.32(0.29)$ & $3.73(0.13)$ \\
& 1 & $0.22(0.01)^{*}$ & $3.37(0.31)^{*}$ & $3.51(0.38)$ \\
& 2 & $0.25(0.04)^{*}$ & $2.12(0.27)$ & $4.29(0.35)^{*}$ \\
elbow & 3 & $0.29(0.09)^{*}$ & $1.84(0.36)$ & $3.99(0.35)$ \\
& 4 & $0.15(0.02)^{*}$ & $2.44(0.28)^{*}$ & $3.87(0.04)$ \\
& 1 & $1.19(0.17)^{*}$ & $1.47(0.23)^{*}$ & $3.16(0.28)$ \\
\multirow{3}{*}{ shoulder } & 2 & $0.91(0.22)$ & $0.89(0.07)^{*}$ & $4.00(0.71)$ \\
& 3 & $1.46(0.19)$ & $0.77(0.12)^{*}$ & $3.85(0.51)$ \\
& 4 & $1.11(0.21)$ & $0.37(0.03)$ & $3.25(0.26)^{*}$ \\
& 1 & $2.24(0.39)^{*}$ & $3.20(0.28)^{*}$ & $3.26(0.45)$ \\
& 2 & $2.00(0.69)$ & $2.93(0.64)$ & $3.63(0.40)$ \\
& 3 & $1.69(0.16)^{*}$ & $1.76(0.22)$ & $2.86(0.13)^{*}$ \\
& 4 & $1.87(0.36)^{*}$ & $4.15(0.36)^{*}$ & $3.62(1.10)$ \\
\hline
\end{tabular}

$* p<0.05$.

Table 3

Overall stiffness (for the wrist, elbow and shoulder) and damping (for the wrist) with and without joint restrictions. The values presented are averages of 5 tests, each over the period of $30 \mathrm{~s}(\mathrm{SD})$

\begin{tabular}{|c|c|c|c|c|c|}
\hline \multirow[t]{2}{*}{ Restriction/joint } & \multirow[t]{2}{*}{ Subject no. } & \multicolumn{2}{|c|}{ Wrist } & \multirow{2}{*}{$\frac{\text { Elbow }}{k(\mathrm{Nm} / \mathrm{rad})}$} & \multirow{2}{*}{$\begin{array}{c}\text { Shoulder } \\
k(\mathrm{Nm} / \mathrm{rad})\end{array}$} \\
\hline & & $k(\mathrm{Nm} / \mathrm{rad})$ & $b(\mathrm{~N} * \mathrm{~m} * \mathrm{sec} / \mathrm{rad})$ & & \\
\hline \multirow[t]{4}{*}{ no } & 1 & $3.358(0.29)$ & $0.562(0.50)$ & $3.028(0.04)$ & $59.684(26.01)$ \\
\hline & 2 & $1.892(0.10)$ & $0.930(0.33)$ & $1.722(0.09)$ & $19.114(0.94)$ \\
\hline & 3 & $2.399(0.51)$ & $0.278(0.62)$ & $2.126(0.07)$ & $39.273(12.59)$ \\
\hline & 4 & $8.447(0.36)$ & $0.155(0.27)$ & $2.157(0.06)$ & $21.393(0.92)$ \\
\hline \multirow[t]{4}{*}{ wrist } & 1 & 40.133 (14.19)* & $0.455(0.79)$ & $2.954(0.07)$ & $54.950(6.33)$ \\
\hline & 2 & $1.436(0.02)^{*}$ & $3.392(3.18)$ & $1.687(0.08)$ & $33.298(3.57)^{*}$ \\
\hline & 3 & $15.545(2.52)^{*}$ & $5.147(4.81)$ & $1.964(0.07)^{*}$ & $49.461(5.06)$ \\
\hline & 4 & $137.690(46.7)^{*}$ & 1.449 (2.9) & $1.983(0.05)^{*}$ & $19.657(0.35)^{*}$ \\
\hline \multirow[t]{4}{*}{ elbow } & 1 & $2.588(0.15)^{*}$ & $1.411(0.66)$ & $4.230(0.16)^{*}$ & $43.167(8.33)$ \\
\hline & 2 & $1.758(0.15)$ & $0.210(0.14)^{*}$ & $3.315(0.05)^{*}$ & $50.809(13.76)^{*}$ \\
\hline & 3 & $1.541(0.13)^{*}$ & $0.999(0.68)$ & $2.525(0.03)^{*}$ & $57.352(7.72)^{*}$ \\
\hline & 4 & $4.247(1.82)^{*}$ & $0.0(0.0)$ & $2.981(0.05)^{*}$ & $19.984(0.48)^{*}$ \\
\hline \multirow[t]{4}{*}{ shoulder } & 1 & $2.955(0.523)$ & $0.592(0.45)$ & $3.576(0.07)^{*}$ & $74.342(26.36)$ \\
\hline & 2 & $7.501(2.36)^{*}$ & $0.751(0.49)$ & $2.024(0.14)^{*}$ & $35.371(13.21)^{*}$ \\
\hline & 3 & $2.102(0.07)$ & $0.974(0.60)$ & $1.715(0.04)^{*}$ & $71.839(11.62)^{*}$ \\
\hline & 4 & $6.383(0.46)^{*}$ & $0.114(0.16)$ & $2.642(0.03)^{*}$ & $52.740(10.41)^{*}$ \\
\hline
\end{tabular}

$* p<0.05$. 
Table 4

Stiffness coeficients $k_{0 j}, k_{2 j}$ values. The values presented are averages of 5 tests, each over the period of $30 \mathrm{~s}$ (SD)

\begin{tabular}{|c|c|c|c|c|c|}
\hline \multirow[t]{2}{*}{ Restriction/joint } & \multirow[t]{2}{*}{ Subject no. } & \multicolumn{2}{|c|}{ Elbow } & \multicolumn{2}{|c|}{ Shoulder } \\
\hline & & $k_{0}(\mathrm{Nm} / \mathrm{rad})$ & $k_{2}\left(\mathrm{~N}^{*} \mathrm{~m}^{*} \mathrm{sec} / \mathrm{rad}^{2}\right)$ & $k_{0}(\mathrm{Nm} / \mathrm{rad})$ & $k_{2}\left(\mathrm{~N}^{*} \mathrm{~m}^{*} \mathrm{sec} / \mathrm{rad}^{2}\right)$ \\
\hline \multirow[t]{4}{*}{ no } & 1 & $3.03(0.04)$ & $1.16(0.94)$ & $59.50(26.1)$ & $-49.43(15.21)$ \\
\hline & 2 & $1.72(0.09)$ & $3.06(0.7)$ & $19.11(0.95)$ & $4.99(8.29)$ \\
\hline & 3 & $2.14(0.07)$ & $-5.63(18.51)$ & 39.15 (12.57) & $-38.50(10.55)$ \\
\hline & 4 & $2.16(0.07)$ & $0.32(0.93)$ & $21.40(0.89)$ & $-9.33(3.91)$ \\
\hline \multirow[t]{4}{*}{ wrist } & 1 & $2.95(0.06)^{*}$ & $2.10(0.96)$ & $54.83(6.27)$ & $-46.26(19.13)$ \\
\hline & 2 & $1.69(0.09)$ & $1.37(1.67)^{*}$ & $33.26(3.54)^{*}$ & $6.50(13.28)$ \\
\hline & 3 & $1.96(0.08)^{*}$ & $-0.59(5.17)$ & $49.41(5.07)$ & $-28.87(8.36)$ \\
\hline & 4 & $1.98(0.05)^{*}$ & $-0.22(1.85)$ & $19.63(5.07)^{*}$ & $-7.64(2.1)$ \\
\hline \multirow[t]{4}{*}{ elbow } & 1 & $4.23(0.16)$ & $5.83(3.13)^{*}$ & $43.13(8.36)$ & $-30.10(10.7)^{*}$ \\
\hline & 2 & $3.31(0.05)^{*}$ & $4.53(1.98)$ & $50.80(13.75)^{*}$ & $-12.92(18.55)^{*}$ \\
\hline & 3 & $1.96(0.03)^{*}$ & $2.28(5.17)$ & $57.30(7.7)^{*}$ & $-31.68(14.02)$ \\
\hline & 4 & $2.98(0.04)^{*}$ & $-4.93(13.77)$ & $19.96(0.49)^{*}$ & $-5.78(2.82)$ \\
\hline \multirow[t]{4}{*}{ shoulder } & 1 & $3.57(0.07)$ & $0.72(3.09)$ & $74.32(26.31)$ & $10.47(18.46)^{*}$ \\
\hline & 2 & $2.02(0.15)^{*}$ & $2.54(2.88)$ & $35.34(13.2)^{*}$ & $-10.75(7.33)^{*}$ \\
\hline & 3 & $1.71(0.04)^{*}$ & $3.03(1.04)$ & $71.85(11.64)^{*}$ & $53.05(18.84)^{*}$ \\
\hline & 4 & $2.64(0.03)^{*}$ & $-0.76(0.93)$ & $52.76(10.45)^{*}$ & $48.17(13.18)^{*}$ \\
\hline
\end{tabular}

$* p<0.05$.

Table 5

Averaged normalized SSE results (normalized by the average value of SSE in case with no restriction). The values presented are averages of 5 tests, each over the period of $30 \mathrm{~s}$

\begin{tabular}{lcccc}
\hline Restriction & Subject no & Wrist & Elbow & Shoulder \\
\hline wrist & 1 & 0.61 & 0.51 & 0.53 \\
& 2 & 5.26 & 9.24 & 1.74 \\
& 3 & $0.84^{*}$ & $0.88^{*}$ & 1.26 \\
elbow & 4 & $1.33^{*}$ & 0.98 & $0.87^{*}$ \\
& 1 & $0.35^{*}$ & 0.27 & $0.4^{*}$ \\
& 2 & $4.16^{*}$ & $7.76^{*}$ & $2.04^{*}$ \\
shoulder & 3 & 0.74 & 1.01 & 1.1 \\
& 4 & $0.83^{*}$ & $1.3^{*}$ & $1.2^{*}$ \\
& 1 & $0.41^{*}$ & 0.43 & 0.72 \\
& 2 & $24.2^{*}$ & $41.2^{*}$ & 29.55 \\
& 3 & $0.67^{*}$ & $0.68^{*}$ & 0.82 \\
& 4 & $1.49^{*}$ & $1.47^{*}$ & $1.38^{*}$ \\
\hline
\end{tabular}

$* p<0.05$

is presented in Table 2. The values designate averages of 5 tests, each over the period of $30 \mathrm{~s}$. The 'no restriction' case served as a reference. The statistically significant effects, marked by asterices, are dominantly seen along the main diagonal of joint restrictions and express the decrease in oscillation amplitudes in each of the restricted joint. It may be noted that each of the wrist, elbow and shoulder limitations caused consistent decreases in the self oscillations of each of these joints, although not all the decreases were statistically significant.

Noticeable out-of-diagonal changes include wrist and elbow oscillatory movements due to shoulder restriction, and elbow oscillatory movements due to wrist restriction. It should be noticed that cross-talk effects could either be of decrease, or increase of amplitude. For instance, increased wrist oscillatory movements due to shoulder restriction could suggest compensatory movement to the 'stiffening' in the shoulder.

\subsection{Estimation results}

\subsubsection{Reduction of the model}

By applying the multicollinearity diagnostic criteria and $F$-test, estimation of the model yielded reduced impedance expressions for each joint. The most significant stiffness coefficients $\sum$ (with $p<0.05$ ) were $k_{0}$ and $k_{2}$, for the elbow and shoulder joints and $k 0$ for the wrist joint, Eq. (1). The damping coefficients $\mathrm{b}_{0}$ was significant only in the wrist joint. Thus, it was concluded that reducing the optimal model to a 3-parameter model, with a nonlinearly variable stiffness and a constant damping would be sufficient, as follows:

for the wrist joint:

$$
\begin{aligned}
& k_{j}(\phi)=k_{0 j} \\
& b_{j}(\dot{\phi})=b_{0 j} \\
& M_{t w}=k_{0 w}\left(\phi_{t w}-\phi_{0 w}\right)+b_{0 w}\left(\dot{\phi}_{t w}-\dot{\phi}_{0 w}\right)
\end{aligned}
$$

for the elbow and shoulder joints:

$$
k_{j}(\phi)=k_{0 j}+k_{2 j}\left(\dot{\phi}_{j}-\dot{\phi}_{0 j}\right)
$$




$$
M_{t j}=k_{0 j}\left(\phi_{t j}-\phi_{0 j}\right)+k_{2 j}\left(\dot{\phi}_{t j}-\dot{\phi}_{0 j}\right)\left(\phi_{t j}-\phi_{0 j}\right)
$$

Table 3 presents values of the overall stiffness and damping (Eqs. (7) and (9)) with and without joint restriction. The values constitute averages of 5 tests, each over the period of $30 \mathrm{~s}$. The 'no restriction' case served as a reference for comparisons ( $t$-test), with significance level of $p<0.05$.

During the tests with no restriction, the overall stiffness values were higher in the shoulder joint than in the elbow and wrist joint. Wrist restriction resulted in an increase in stiffness and damping in that joint in 3 out of the 4 subjects. The effect on the elbow stiffness was that of a decrease in all 4 subjects. The effect on the shoulder stiffness was not uniform. Elbow restriction demonstrated a stiffness increase in that joint. The effect on the wrist was a decrease in stiffness. The effect on damping was not uniform. Likewise, the elbow restriction did not cause any uniform effect on the stiffness or damping of the shoulder. Shoulder restriction resulted in an increase in stiffness on that joint. In three subjects this restriction resulted in a decrease in stiffness in the wrist and an increase in stiffness in the elbow.

Table 4 presents values of stiffness coeficients $k_{0 j}, k_{2 j}$, (Eq. (9)) with and without joint restrictions. Here again, the values represent averages of 5 tests, each over the period of $30 \mathrm{~s}$, and the 'no restriction' case served as a reference for comparisons ( $t$-test). Wrist restriction resulted in a decrease in $k_{0}$ and $k_{2}$ of the elbow joint, and a decrease in $k_{2}$ of the shoulder joint. Elbow restriction evoked an increase in and of elbow joint, and a decrease in of the shoulder joint.

Shoulder restriction resulted in an increase in $k_{0}$ and a decrease in $k_{2}$ of the elbow joint, and an increase in $k_{0}$ and $k_{2}$ of the shoulder joint (for comparison of the $k_{2}$ values, absolute values were taken). As seen from Table 4, the effect of shoulder and elbow restrictions was mostly expressed through changes in $k_{0 \mathrm{j}}$. It may also be seen that the stiffness coefficient values of $k_{0}$ and $k_{2}$ were higher for shoulder compared to the other joints.

Comparing the coefficient values at no restriction among the subjects at the different joints (Tables 3,4), we note that the values of $k_{0}$ of the wrist range within $1.9-8.5 \mathrm{Nm} / \mathrm{rad}$. For $b_{0}$ the range is $0.1-0.9 \mathrm{Nms} / \mathrm{rad}$. In the elbow the $k_{0}$ range is $1.7-3 \mathrm{Nm} / \mathrm{rad}$ and that of $k_{2}$ is $0.3-5.6 \mathrm{Nms} / \mathrm{rad}^{2}$. The overall stiffness in this joint ranges $1.7-3 \mathrm{Nm} / \mathrm{rad}$. For the shoulder joint the $k_{0}$ values are found to be within $19.1-59.5 \mathrm{Nm} / \mathrm{rad}$ and those of $k_{2}$ within $4.9-49.4 \mathrm{Nms} / \mathrm{rad}^{2}$ (absolute values). The overall stiffness in the shoulder joint ranged within $19.1-59.7 \mathrm{Nm} / \mathrm{rad}$. $k_{2}$ had a negative value in most cases.

Statistical analysis of SSE value for verifying the accuracy of the obtained impedance model included normalizing SSE values by mean SSE value from tests without limitation. The results indicated that the accuracy changed (higher and lower values) for different limitations (Table 5). As seen from this table, shoulder limitation caused in $75 \%$ of the cases the greatest effect $(p<0.05)$ on the values of the other two joints: wrist and elbow. This was not the case in the limitation of either the elbow or wrist joints. In subject \#2 the greatest increase in SSE values was during tests with limitation.

Sensitivity tests showed that the changes in parameters $k_{0}, k_{2}$, and $b_{0}$ and SSE values were smaller than $25 \%$ for the maximal changes of $20 \%$ in measured angles, mass of segments and moment of inertia. For the maximal (20\%) changes in the horizontal position of the shoulder SSE values in the elbow model were smaller than $46 \%$.

\section{Discussion}

The task of steadily carrying a cup with liquid while walking is an interesting motor control task. The biomechanical configuration examined in this study included the cup as the 'end-effector' of a serial 3-link chain representing the upper limb, attached to the carrier base (body) via the shoulder girdle. The system was assumed to move in the sagittal plane due to the motion of each of the shoulder, elbow and wrist joints about the medio-lateral axis and this motion was monitored. The experimental setting was in the steady state of walking on a constant-speed treadmill, which forces the system into a single frequency, in accordance with the walking stride. In practice, the oscillatory motion of the upper limb holding the cup is not a purely harmonic motion, and instead of a single frequency, a narrow band of concurent oscillations would result for this motion around this frequency.

Clearly, the characterization of the system could be expanded to a larger spectrum of frequencies if other modes of motion including step-response input to the upper limb or input due to standing on a randomly movable support would have replaced steady walking. Despite this limitation, the present approach allows to 
phenomenologically describe joint impedances of the upper limb in a typical physiological daily activity such as walking while fulfilling the minimum liquid spillage target function. The effect of successive immobilization of each of the joints on the impedances was also studied. In a precise sense, the joints were restricted rather than fully immobilized, due to the fact that the splints used were not fully rigid and the splint-body interface contained the somewhat compliant soft tissue layer.

Although coordination between locomotion and control has been studied in the past [2, 4, 30], no works were found dealing with the simultaneous control of grasping and walking when joints of the upper limb were constrained. We used here the liquid level to indicate how well the limb navigated itself to minimize liquid spillage from the instrumented cup and how the limb adapted to the joint restrictions that were imposed. The three level markings on the cup served to monitor the extent to which the target function was fulfilled, the highest indicating the greatest amount of liquid spillage. In all four subjects, the liquid level did not reach the third and highest level during steady-state motion. This indicated that the subjects succeeded in stabilizing their end-effector, irrespective of whether or not restrictions were applied to the joints.

Stiffness regulation of the upper limb joints was reported to depend upon posture configuration $[17,20]$. Thus, upon examining the motion strategies adopted by the test subjects and their effect on joint impedances, it is interesting to note that the postural configuration of the shoulder joint (Table 1); in three of the subjects the upper arm was sagittaly positioned in front of the vertical line, while in one subject (number 2) the upper arm was positioned behind the vertical and was stayed so throughout the experiments. Indeed, the stiffness coefficients were the lowest in this subject, particularly in those tests where no restrictions were imposed.

Imposing restrictions proved to affect the postural configuration of the restricted joint as well as the unrestricted joints (Table 1).

The muscle-tendon complex is capable of storing, releasing and absorbing energy and its stiffness generally depends on the activation level of the muscle. Although it can also generate motion and/or force to oppose an external disturbance, this effect was overlooked in the present study because the motion studied was in steady state mode and external disturbances were not expected. The basic model was thus made to include elastic and damping elements. The elastic element depended on angular displacement and angular velocity [32] and the damping element depended on angular velocity [16]. By testing multicollinearity, this model was reduced and adapted separately to each of the joints. Tregoubov [28] discussed the non-uniqueness of impedance representation: different parameter representations of mechanical impedance may result in the same joint behavior. Seeking for as much uniform behavior as possible, we implemented the model reduction obtained in this study for each joint to all four subjects. The downside of that is that high-quality match cannot be expected in all experiments. Obviously, increasing of the number of tested subjects would have improved the model obtained for each of the joints.

The wrist joint was found to have constant stiffness and damping (Eq. 7), and no regulation of these coefficients was necessary during the gait cycle. It should be reminded that the finger joints were not represented in the end-effector and this segment was considered a rigid body attached to the wrist joint. The other two joints had non-linear representations (Eq. 9). Non-linear models are widespread in the description of human joints $[7,11,12,21,22]$. In both the elbow and shoulder joints, stiffness included a constant coefficient as well as an angular velocity-dependent coefficient. It should be noted here that no distinction could be made between angular velocity-dependency of stiffness and angle-dependency of damping due to their similar effect on the joint torque, and estimation was based on the difference between the moments in Equation (6). Thus, a damping term per se was found unnecessary.

Examining the oscillatory amplitudes of the joint angles (Table 2) revealed that wrist oscillations were the smallest compared to the other joints. This should not be surprising because distal joints provide the refined adjustment of hand motion whereas the more proximal ones dominate global navigation of the hand Levin et al. [15]. Restricting a joint decreased the joint oscillations of that joint and increased its stiffness.

The effect of joint restrictions on the goodness of fit of the model of the same as well as of the other joints, as assessed by means of SSE, was not uniform. Shoulder restriction seemed to have the greatest effect on the SSE of estimation for all three joint models with the highest level of significance for the wrist and elbow joints (Table 5). For two of the subjects $(\# 2,4)$ there was an increase in SSE $(>1)$, while for the other two $(\# 1,3)$ there was a decrease in SSE $(<1)$. 
A comment should be made to address possible effects of learning and/or fatigue over the time of conducting the experiments. Learning was reported to decrease joint total stiffness [26]. In the present study we assumed that, following the warm-up phase before measurements began; the tested subjects already had developed a steady pattern that did not significantly change during the subsequent five $30 \mathrm{~s}$-tests and that subsequent learning and fatiguing were negligible.

This study sheds light on the mechanisms of stabilization of manually held objects during walking. All the four subjects who took part demonstrated the ability to stabilize the cup of liquid despite the restrictions successively imposed on the joints. The wide variability in the impedance results obtained indicated that the compensatory mechanisms exercised by each subject to regulate the mechanical impedance so as to respond to the joint restriction were individual, and not necessarily a common strategy. Nevertheless, these results may pertain to future designing of artificial arms and robotic devices and to the development of more accurate control strategies.

It may be of interest to compare stiffness and damping ranges obtained in this study (Table 3 ) with values found in the literature for upper limb joints. Such comparison should, however, be treated with caution viewing that impedance coefficients depend on the models used to represent the limb and the joint, and the characteristics of the loads acting. For the elbow Hayes and Hatze [7] found that the passive stiffness element of ranges within $1.0-1.4 \mathrm{Nm} / \mathrm{rad}$ and the damping values range within $0.057-0.274 \mathrm{Nms} / \mathrm{rad}$ for a motion range within $0.56-1.55 \mathrm{rad}$. Flash and Gurevich [3] reported an elbow stiffness ranging within $6.9-117.0 \mathrm{Nm} / \mathrm{rad}$ depending on position, for a group of four subjects. $\mathrm{Xu}$ and Hollerbach [33] reported peak values of $90 \mathrm{Nm} / \mathrm{rad}$ and 1.7 Nms/rad for stiffness and damping, respectively. For the shoulder joint Zhang et al. [35] reported stiffness values within $30-180 \mathrm{Nm} / \mathrm{rad}$. For the wrist joint Milner and Cloutier [15] reported peak values of $6 \mathrm{Nm} / \mathrm{rad}$ and $0.07 \mathrm{Nms} / \mathrm{rad}$ for stiffness and damping, respectively.

A limitation of this study is that the end-effector was assumed to be a rigid body; hence the finger joints were neglected. There could have been some contributions of additional motion of the fingers in the stabilization of the grasped object. Another limitation is the confinement to sagittal plane motion, while the monitored liquid spillage was actually three dimensional. A final comment relates to the sample size of the tested sub- jects. Clearly, increasing the sample size would help establishing the consistency of the results obtained. Future studies will have to address these issues.

\section{Acknowledgments}

The experiments in this study were part of a Master of Science project of first author NR and were performed in the Biomechatronics Laboratory in the Technion Biomedical Engineering Department. This work was supported in part by funds from the Technion's Lady Davis Foundation, and the Stein fellowship Fund at Drexel University.

\section{References}

[1] C.P. Bertram, R.G. Marteniuk and D.C. Mackey, Increasing upper limb task complexity leads to gait adaptations, Canadian Society for Psychomotor Learning and Sport Psychology, SCAPPS, Waterloo, 2000, p. 47.

[2] H. Carnahan, B.J. McFadyen, D.L. Cockell and A.H. Halverson, The combined control of locomotion and prehension, Neurosci Res Commun 19 (1996), 91-100.

[3] T. Flash and I. Gurevich, Models of motor adaptation and impedance control in human arm movements, In P. Morasso and V. Sanguineti (eds), Self Organization, Computational Maps, and Motor Control Elsevier Science BV, Amsterdam, 1997, pp. 423-481.

[4] A.P. Georgopoulos and S. Grillner, Visuomotor coordination in reaching and locomotion, Science 245 (1989), 1209-1210.

[5] H. Gomi and R. Osu, Task dependent viscoelasticity of human multijoint arm and its spatial characteristics for interaction with environments, J of Neurosc 18(21) (1998), 8965-8978.

[6] R. Grasso, M. Zago and F. Lacquaniti, Interactions between posture and locomotion: motor patterns in humans with bent versus with erect posture, J Neurophysiol 83 (2000), 288-230.

[7] K.C. Hayes and H. Hatze, Passive viscoelastic parameters of the structures spanning the human elbow joint, Europ J Appl Physiol 37 (1977), 265-274.

[8] N. Hogan, Mechanical impedance of single-and multiarticular systems, In J.M. Winters and S.L-Y. Woo (eds), Multiple Muscle Systems: Biomechanics and Movement Organization, Springer-Verlag, New York, 1990, pp. 149-164.

[9] P.Ch. Ivanov, Q.D.Y. Ma, R.P. Bartsch, J.M. Hausdorff, L.A.N. Amaral, V. Schulte- Frohlinde, H.E. Stanley and M. Yoneyama Levels of complexity in scale-invariant neural signals. Phys. Rev. E 79, (2009) 041920 [12 pages].

[10] T.R. Kane, D.A. Levinson, Dynamics: Theory and Application, McGraw-Hill Book Company, New York, 1985

[11] A. Karniel and G.F. Inbar, A model of learning human reaching movements, Biol Cybern 77 (1997), 173-183.

[12] A. Karniel and G.F. Inbar, The use of nonlinear muscle model in explaining the relatioship between duration, amplitude, and peak velocity of human rapid movements, J Motor Behav 31(3) (1999), 203-206. 
[13] J. Konczak, K. Brommann and K.T. Kalveram, Identification of time varying stiffness, damping and equilibrium position in human forearm movements, Motor control 3 (1999), 394-413.

[14] F. Lacqaniti, M. Carrozzo and N.A. Borghese, Time varying mechanical behavior of multijointed arm in man, $J$ Neurophysiol 69(5) (1993), 1443-1464.

[15] O. Levin, M. Ouamar, M. Steyvers and S.P. Swinnen, Directional tuning effects during cyclical two-joint arm movements in the horizontal plane, Exp Brain Res 141 (2001), 471-484.

[16] T.E. Milner and C. Cloutier, Damping of the wrist joint during voluntary movement, Exp Brain Res 122 (1998), 309-317.

[17] T.E. Milner, Contribution of geometry and joint stiffness to mechanical stability of the human arm, Exp Brain Res 143 (2002), 515-519.

[18] F.A. Mussa-Ivaldi, N. Hogan and E. Bizzi, Neural, mechanical and geometric factors subserving arm posture in humans, $J$ Neurosc 5(10) (1985), 2732-2743.

[19] A.E. Patla, M.G. Ishac and D.A. Winter, Anticipatory control of center of mass and joint stability during arm movements from a standing posture: interplay between active and passive control, Exp Brain Res 143 (2002), 318-327.

[20] E.J. Perreault, R.F. Kirsch and P.E. Crago, Voluntary control of static endpoint stiffness during force regulation tasks, $J$ Neurophysiol 87 (2002), 2808-2816.

[21] S. Rakheja, R. Gurram and G.J. Gouw, Development of linear and nonlinear hand-arm vibration models using optimization and linearization techniques, J Biomech 26(10) (1993), 1253-1260.

[22] S. Rapoport, J. Mizrahi, E. Kimmel, O. Verbitsky and E. Isakov, Constant and variable stiffness and damping of the leg joints in human hopping, J Biomech Eng 125 (2003), 507-514.

[23] J.B. Reswick, Development of feedback control prosthetic and orthotic devices, In J.H.U. Brown and J.F. Dickson (eds), Advances in Biomedical Engineering, vol 2. Academic Press London, 1972, pp. 139-217.

[24] D.D. Reynolds and W. Soedel, Dynamic response of the hand-arm system to sinusoidal input, In W. Taylor (ed), The Vibration Syndrome, Academic Press, London, 1974, pp. 149-168.

[25] D.A. Rosenbaum, R.G. Meulenbroek, J. Vaughan and C. Jansen, Posture-based motion planning: applications to grasping, Psychol Rev 108 (2001), 709-773.
[26] S. Stroeve, Impedance characteristics of neuromusculoskeletal model of the human arm, I posture control Biol Cybern 81 (1999), 475-494.

[27] C.W. Suggs, Modelling of the dynamic characteristic of the hand-arm system, In W. Taylor (ed.), The Vibration Syndrome, Academic Press London, 1974, pp. 169-186

[28] V.P. Tregoubov, Problems of mechanical model identification for human body under vibration, Mechanism and Machine Theory 35 (2000), 491-504.

[29] T. Tsuji, Human arm impedance in multi-joint movements, In P. Morasso and V. Sanguineti (eds), Self Organization, Computational Maps, and Motor Control, Elsevier Science B.V., Amsterdam, 1997, pp. 357-382.

[30] R.P.R.D. Van der Wel and D.A. Rosenbaum, Coordination of locomotion and prehension, Exp Brain Res 176 (2007) 281-287.

[31] T. Wang, G.S. Dordevic and R. Shadmehr, Learning the dynamics of reaching movements results in the modification of arm impedance and long latency perturbation responses, Biol Cybern 85 (2001), 437-448.

[32] S.L.Y. Woo and E.P. Young, Structure and function of tendons and ligaments, In V.C. Mow and W.C. Hayes (eds) Basic Orthopedic Biomechanics, 2th edition, Raven Press, New York, 1991, pp. 199-243

[33] Y. Xu and J.M. Hollerbach, Identification of human joint mechanical properties from single trial data, IEEE Trans Biomed Eng 45(8) (1998), 1051-1059.

[34] F.E. Zajac and J.M. Winters, Modelling muscle system- joint and body segmental Dynamics'musculoskeletal actuation, and neuromuscular control, In J.M Winters and S.L-Y Woo (eds), Multiple Muscle Systems : Biomechanics and Move ment Organization, Springer-Verlag, New York, 1990, pp. 121-148.

[35] L.Q. Zhang, G.H. Portland, G. Wang, C.A. Diraimondo, G.W Nuber, M.K. Bowen and R.W. Hendrix, Stiffness, viscosity and upper limb inertia about the glenohumeral abduction axis, Journal Orthop Res 18 (2000), 94-100.

[36] M.B. Popovic, D.B. Popovic and R. Tomovic, Control of arm movement: reaching synergies. J Aut Control 12 (2002), 9-15. 

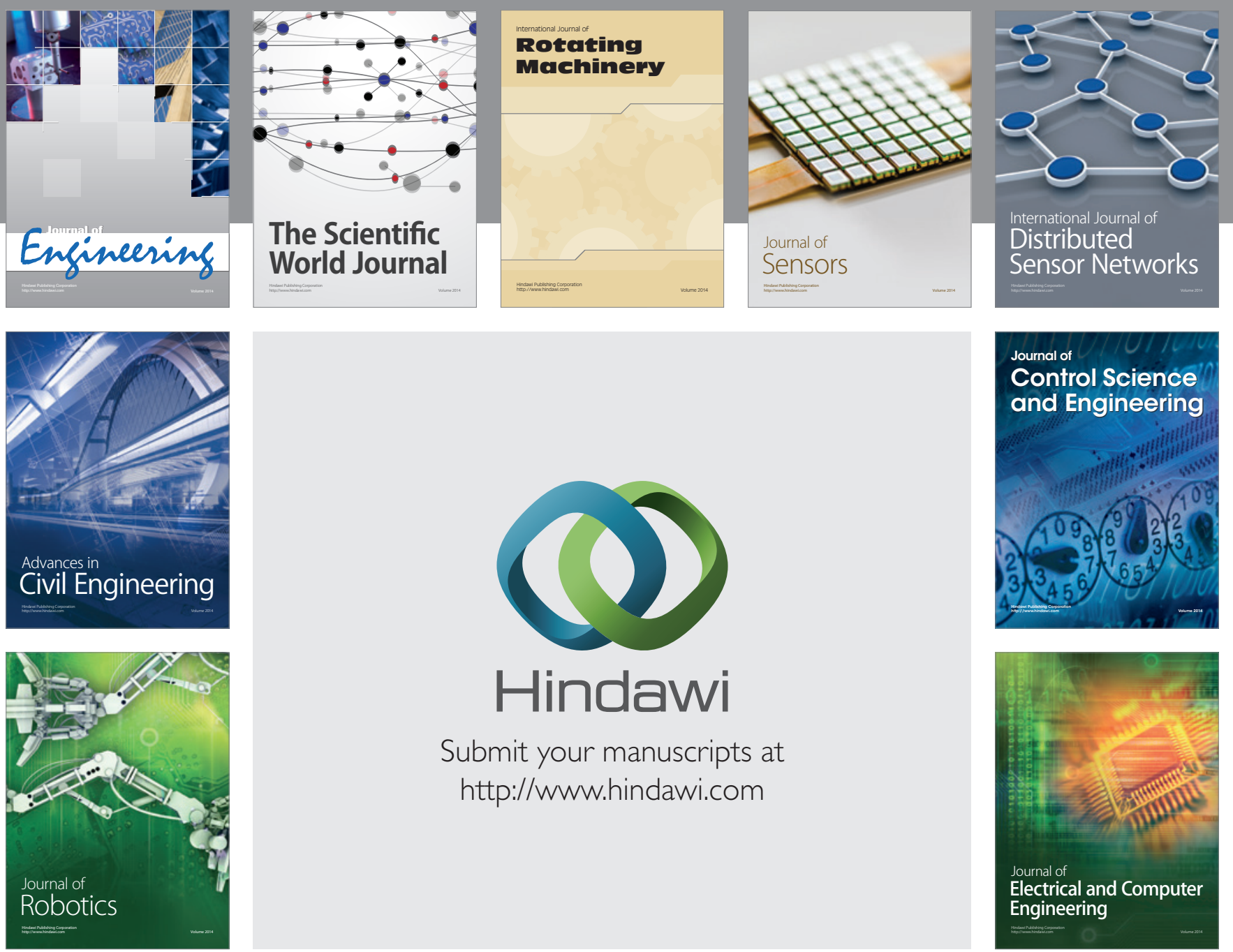

Submit your manuscripts at

http://www.hindawi.com
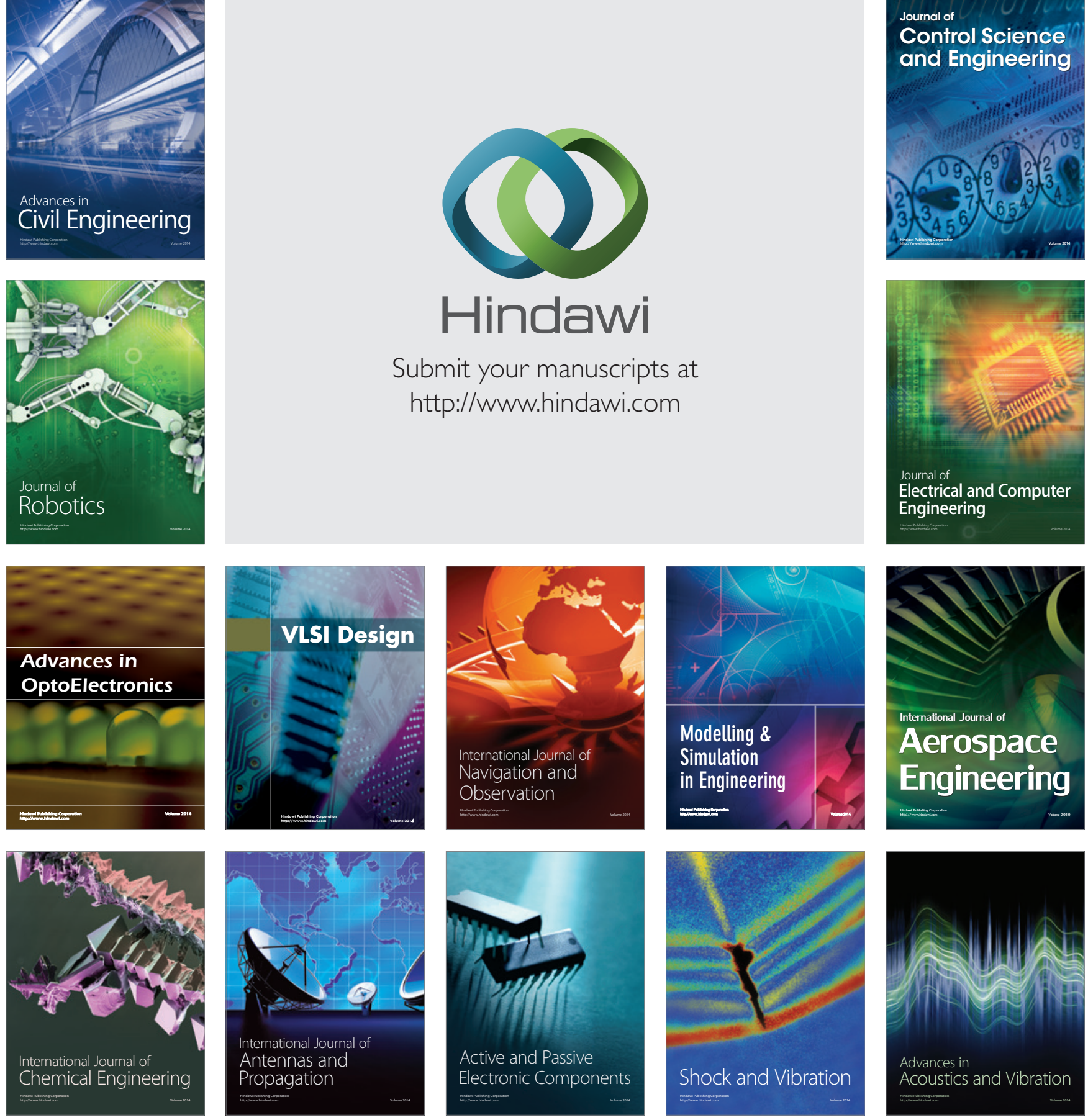